\title{
CHANNEL ESTIMATION AND SYNCHRONIZATION WITH SUB-NYQUIST SAMPLING AND APPLICATION TO ULTRA-WIDEBAND SYSTEMS
}

\author{
Irena Maravić ${ }^{\dagger}$, Martin Vetterli ${ }^{\dagger \ddagger}$ and Kannan Ramchandran ${ }^{\ddagger}$ \\ ${ }^{\dagger}$ Audio-Visual Communications Laboratory \\ Swiss Federal Institute of Technology in Lausanne, CH-1015 Lausanne, Switzerland \\ ${ }^{\ddagger}$ Department of EECS, University of California at Berkeley, Berkeley CA 94720, USA
}

\begin{abstract}
We consider the problem of low-complexity channel estimation and timing in digital ultra-wideband receivers. We extend some of our recent results on sampling of certain classes of parametric nonbandlimited signals and develop a frequency domain framework that yields high-resolution estimates of all relevant channel parameters by sampling a received signal below the traditional Nyquist rate. In particular, we show that the minimum required sampling rate in an UWB receiver is determined by the so-called innovation rate, which corresponds to the number of degrees of freedom of the received UWB signal. Our framework allows for faster acquisition compared to current digital solutions and potentially reduces power consumption and complexity of digital UWB receivers significantly. It is particularly suitable in applications such as ranging or positioning and can also be used for identification of more realistic UWB channel models, where different propagation paths undergo different frequency-selective mitigation.
\end{abstract}

\section{INTRODUCTION}

Ultra-wideband (UWB) technology has recently received much attention as a potential solution to a variety of short-range problems, such as accurate ranging and positioning as well as multipath fading mitigation in indoor wireless networks [1] [4]. UWB systems use trains of pulses of very short duration, typically on the order of a nanosecond, thus spreading the signal energy from near DC to a few gigahertz. Although a possibility of using extremely short pulses has been investigated for at least two decades, primarily in the radar community, there still remains a lot to be done for this technology to become pervasive. Some of the important issues include low-cost and low-power designs and efficient algorithmic solutions suitable for digital implementation.

The nature of UWB signaling brings new challenges both in the analysis and practice of reliable systems. One of the main design challenges is rapid synchronization, as its accuracy and complexity directly affect the system performance [4]. This problem becomes even more involved with a current trend to minimize the number of analog components needed, and perform as much processing digitally as possible [2]. Namely, due to the extreme bandwidths involved, digital implementation may lead to prohibitively high costs in terms of power consumption and receiver complexity. For example, conventional techniques based on sliding correlators would require very fast and expensive A/D converters (operating in the gigahertz range) and, therefore, high power consumption. Furthermore, implementation of such techniques in digital systems would have almost unaffordable complexity as well as slow convergence time, since one has to perform exhaustive search over thousands of fine bins, each at the nanosecond level. In order to improve the acquisition speed, several modified timing recovery schemes have been proposed, such as a bit reversal search [3], or the correlator-type approach which exploits properties of beacon sequences [4]. Even though some of these methods have already been in use in certain analog systems, the need for very high sampling rates, along with the search-based nature of such methods, makes them less attractive for digital implementation.

In this paper, we present a new approach to channel estimation and timing in digital UWB receivers, which allows for sub-Nyquist sampling rates and reduces the receiver complexity, while retaining a desirable performance. The idea is based on our recent results on sampling of certain classes of parametric non-bandlimited signals that have a finite number of degrees of freedom per unit of time, or a finite rate of innovation. We show that the minimum required sampling rate in UWB systems is determined by the innovation rate of the received UWB signal [5] [6], rather than the Nyquist rate. Our approach allows for lower sampling rates and reduced complexity and power consumption compared to other digital techniques [2] [3]. It is particularly suitable in applications such as ranging or positioning, but can also be used for estimation of more general UWB channels [1].

\section{LOW-SAMPLING RATE PARAMETER ESTIMATION}

\subsection{Problem Statement}

A number of propagation studies for UWB signals have been done, which take into account temporal properties of a channel or characterize a spatio-temporal channel response [1]. A typical model for the impulse response of a multipath fading channel is given by

$$
h(t)=\sum_{l=1}^{L} a_{l} \delta\left(t-t_{l}\right),
$$

where $t_{l}$ denotes a signal delay along the $l$-th path, while $a_{l}$ is a complex propagation coefficient. Equation (1) implies that a received signal $y(t)$ is made up of a sum of attenuated and delayed replicas of a transmitted signal $s(t)$, i.e.

$$
y(t)=\sum_{l=1}^{L} a_{l} s\left(t-t_{l}\right)+\eta(t),
$$


where $\eta(t)$ denotes receiver noise. Let $Y(\omega)$ denote the Fourier transform of the received signal

$$
Y(\omega)=\sum_{l=1}^{L} a_{l} S(\omega) e^{-j \omega t_{l}}+\mathcal{N}(\omega),
$$

where $S(\omega)$ and $\mathcal{N}(\omega)$ are Fourier transforms of $s(t)$ and $\eta(t)$ respectively. Clearly, spectral components are given by a sum of complex exponentials, thus we can convert the problem of estimating the unknown channel parameters $\left\{t_{l}\right\}_{l=0}^{L-1}$ and $\left\{a_{l}\right\}_{l=0}^{L-1}$ into the classic spectral estimation problem. High-resolution spectral estimation is well-studied: there exists a rich body of literature on efficient algorithms and achievable performance bounds [7]. In the following, we will adopt a model-based approach and show how it can be used to obtain high-resolution estimates of all the relevant parameters from a subsampled version of the received signal.

\subsection{Polynomial Realization of the Model-Based Estimator}

Suppose that the received signal $y(t)$ is filtered with an ideal bandpass filter $H_{b}=\operatorname{rect}\left(\omega_{1}, \omega_{2}\right)$, and sampled uniformly at a rate $R_{s} \geq \frac{\omega_{2}-\omega_{1}}{2 \pi}$. Suppose next that $N$ uniformly spaced frequency samples of $Y(\omega)$ are available, that is,

$$
Y[n]=Y\left(\omega_{n}\right)=Y\left(\omega_{1}+(n-1) \omega_{0}\right), \quad n=1, \ldots, N .
$$

where $\omega_{0}=\frac{\omega_{2}-\omega_{1}}{N-1}$ and let $Y_{s}[n]=Y[n] / S[n]$, where $S[n]$ are the samples of the Fourier transform $S\left(\omega_{n}\right)$ of the transmitted UWB pulse. Assuming that in the considered frequency band the above division is not ill-conditioned, the samples $Y_{s}[n]$ can be expressed as a sum of complex exponentials (3), i.e.,

$$
Y_{s}[n]=\sum_{l=1}^{L} a_{l} e^{-j \omega_{n} t_{l}}+\mathcal{N}\left(\omega_{n}\right) .
$$

The polynomial approach exploits the fact that in the absence of noise, each exponential $\left\{e^{-j n \omega_{0} t_{l}}\right\}_{n \in Z}$ can be "nulled out" or annihilated by a first order FIR filter $H_{l}(z)=\left(1-e^{-j \omega_{0} t_{l}} z^{-1}\right)$, that is,

$$
e^{-j n \omega_{0} t_{l}} *\left[1,-e^{-j \omega_{0} t_{l}}\right]=0 .
$$

Consider thus an $L$-th order FIR filter $H(z)=\sum_{m=0}^{L} H[m] z^{-m}$, having $L$ zeros at $z_{l}=e^{-j \omega_{0} t_{l}}$,

$$
H(z)=\prod_{l=1}^{L}\left(1-e^{-j \omega_{0} t_{l}} z^{-1}\right)
$$

Note that $H[m]$ is the convolution of $L$ elementary filters with coefficients $\left[1,-e^{-j \omega_{0} t_{l}}\right], l=1, \ldots L$. Since $Y_{s}[n]$ is the sum of complex exponentials, each will be annihilated by one of the roots of $H(z)$, thus we have:

$$
\left(H * Y_{s}\right)[n]=\sum_{k=0}^{L} H[k] Y_{s}[n-k]=0, \quad n=L+1, \ldots, N .
$$

In matrix form, the system (8) is equivalent to

$$
\left(\begin{array}{cccc}
Y_{s}[L+1] & Y_{s}[L] & \cdots & Y_{s}[1] \\
Y_{s}[L+2] & Y_{s}[L+1] & \ldots & Y_{s}[2] \\
\vdots & \vdots & \ddots & \\
Y_{s}[2 L] & Y_{s}[2 L-1] & \cdots & Y_{s}[L] \\
\vdots & \vdots & &
\end{array}\right) \cdot\left(\begin{array}{c}
H[0] \\
H[1] \\
\vdots \\
H[L]
\end{array}\right)=\mathbf{0 .}
$$

By setting $H[0]=1$, one can uniquely solve for $H[m], m=$ $1, \ldots, L$, from only $2 L$ equations in (9). Therefore, the information about the delays $t_{l}$ can be extracted from the roots of $H(z)$, while the corresponding coefficients $a_{l}$ are then estimated by solving the system of linear equations (5), by fitting $L$ exponentials $e^{-j \omega_{n} t_{l}}$ to the data set $Y_{s}[n]$.

The above result can be interpreted in the following way: the signal $y(t)$ is projected onto a low-dimensional subspace corresponding to its bandpass version. This projection is a unique representation of the signal as long as the dimension of the subspace is greater than or equal to the number of degrees of freedom. That is, since $y(t)$ has $2 L$ degrees of freedom, $\left\{t_{l}\right\}_{l=0}^{L-1}$ and $\left\{a_{l}\right\}_{l=0}^{L-1}$, it suffices to use only $2 L$ adjacent coefficients $Y_{s}[n]$.

\subsection{Algorithm in the Presence of Noise: Subspace Approach}

In the case of noiseless data, any subspace of sufficient dimension can be used for estimation. In practice, noise will be present, and this can be dealt with by oversampling and using standard techniques from noisy spectral estimation, such as the singular value decomposition (SVD). Besides, in the presence of noise, it is desirable to estimate the channel from a frequency band where the signal-to-noise ratio (SNR) is highest. This brings us to a more practical version of the model-based approach, the so-called subspace or SVD-based estimator, which avoids the root finding step and relies only on a right deployment of matrix manipulations [6].

Namely, the key is to observe two properties of the matrix $\mathbf{Y}_{\mathbf{S}}$ in (9). The first one is that in the case of noiseless data, $\mathbf{Y}_{\mathbf{S}}$ has rank $L$. This allows us to reduce the noise level by approximating a noisy data matrix with a rank $L$ matrix, by computing its SVD, i.e, $\mathbf{Y}_{\mathbf{s}}=\mathbf{U}_{\mathbf{s}} \boldsymbol{\Lambda}_{\mathbf{s}} \mathbf{V}_{\mathbf{s}}{ }^{H}+\mathbf{U}_{\mathbf{n}} \boldsymbol{\Lambda}_{\mathbf{n}} \mathbf{V}_{\mathbf{n}}{ }^{H}$, where $\mathbf{U}_{\mathbf{s}}$ and $\mathbf{V}_{\mathbf{s}}$ contain principal left and right singular vectors of $\mathbf{Y}_{\mathbf{s}}$. The second property is the Vandermonde structure of $\mathbf{U}_{\mathbf{s}}$ and $\mathbf{V}_{\mathbf{s}}$, that is, they both satisfy the so-called shift-invariant subspace property [6],

$$
{\overline{\mathbf{U}_{\mathbf{s}}}}^{d}={\underline{\mathbf{U}_{\mathbf{s}}}}_{d} \cdot \boldsymbol{\Phi} \text { and }{\overline{\mathbf{V}_{\mathbf{s}}}}^{d}=\underline{\mathbf{V}}_{\mathbf{s}_{d}} \cdot \boldsymbol{\Phi}^{H}
$$

where $\boldsymbol{\Phi}$ is a diagonal matrix having elements $e^{j \omega_{0} d t_{l}}=e^{j \omega_{0} \hat{t}_{l}}$ along the main diagonal, while $\overline{(\cdot)} d$ and $(\cdot)$ denote the operations of omitting the first $d$ rows and the last $\frac{d}{d}$ rows of $(\cdot)$ respectively. Therefore, the time delays $\left\{t_{l}\right\}_{l=1}^{L}$ can be uniquely determined from the eigenvalues $\lambda_{l}$ of the operator that maps $\underline{\mathbf{U}}_{\mathbf{s}}$ onto $\overline{\mathbf{U}}_{\mathbf{s}}^{d}$ (or $\underline{\mathbf{V}}_{\mathbf{s}}$ onto ${\overline{\mathbf{V}_{\mathbf{s}}}}^{d}$ ), that is, $t_{l}=\frac{N \angle \lambda_{l}}{2 \pi d}$. The advantage of using values of $d$ larger than $d=1$, is that the separation between the estimated time delays $\hat{t}_{l}$ is effectively increased $d$ times, which is of particular interest in the case of estimating closely spaced dominant components in a low SNR regime [6]. Once the time delays have been estimated, the coefficients $a_{l}$ can be found from the Vandermonde system (5). Finally, we note that the number of dominant components is estimated as the number of dominant singular values of $\mathbf{Y}_{\mathbf{s}}$.

A major computational requirement of the above method is associated with the SVD step, which is an iterative algorithm with the computational order of $\mathcal{O}\left(N^{3}\right)$ per iteration. In [6], we suggested alternative methods of lower complexity, such as the Power method and the method of Orthogonal iteration. These methods have computational requirements on the order of $\mathcal{O}\left(N^{2}\right)$ per iteration, and they generally converge in less than 10 iterations. 


\section{EXTENSIONS AND SOME APPLICATIONS}

\subsection{Estimating More Realistic UWB Channel Models}

Our frequency-domain framework can be extended to handle the more complex case of an UWB channel that takes into account certain bandwidth-dependent properties [1]. Namely, as a result of the very large bandwidth of UWB signals, components propagating along different propagation paths undergo different frequency selective distortion and a more realistic UWB channel model is of the form

$$
h(t)=\sum_{l=1}^{L} a_{l} p_{l}\left(t-t_{l}\right),
$$

where $p_{l}(t)$ are different pulse shapes that correspond to different paths. In this case, the spectral coefficients of the received signal are given by

$$
Y[n]=S[n] \sum_{l=1}^{L} P_{l}[n] a_{l} e^{-j \omega_{n} t_{l}}+\mathcal{N}[n],
$$

where $P_{l}[n]$ are now unknown coefficients. One possible way to obtain a closed form solution is to approximate the coefficients $P_{l}[m]$ with polynomials of degree $D \leq R-1$, that is,

$$
P_{l}[n]=\sum_{r=0}^{R-1} p_{l, r} n^{r} .
$$

Equation (12) now becomes

$$
Y[n]=S[n] \sum_{l=1}^{L} a_{l} \sum_{r=0}^{R-1} p_{l, r} n^{r} e^{-j \omega_{n} t_{l}}+\mathcal{N}[n] .
$$

By denoting $c_{l, r}=a_{l} p_{l, r}$ and $Y_{s}[n]=Y[n] / S[n]$, we obtain

$$
Y_{s}[n]=\sum_{l=1}^{L} \sum_{r=0}^{R-1} c_{l, r} n^{r} e^{-j \omega_{n} t_{l}}+\mathcal{N}[n] .
$$

In this case, the shift-invariance property no longer holds, however, one can prove that a filter with multiple roots at $z_{l}=e^{-j \omega_{0} t_{l}}$, i.e.

$$
H(z)=\prod_{l=1}^{L}\left(1-e^{-j \omega_{0} t_{l}} z^{-1}\right)^{R}=\sum_{k=0}^{R L} H[k] z^{-k}
$$

will be the annihilating filter for $Y_{s}[n]$ [6]. Therefore, the information about the time delays $t_{l}$ can be extracted from the roots of the filter $H(z)$. The corresponding pulse shapes are then estimated by solving for the coefficients $c_{l, r}$ from the system (15).

In previous work [1], Cramer, Win and Scholtz used an array of sensors to spatially separate the multipath components, which is then followed by identification of each path using the so-called Sensor-CLEAN algorithm. Note that our approach does not require an antenna array, and takes advantage of the fact that the multipath components will have independent delays at the reception [6].

\subsection{Low-Complexity Rapid Acquisition in UWB localizers}

One of the most appealing applications of our framework can be found in ultra-wideband systems used for precise position location. Such UWB transceivers use low duty-cycle periodic transmission of a coded sequence of impulses to insure low-power operation [4]. Yet, rapid synchronization presents a major design challenge, and current systems still rely on exhaustive search through all possible code positions. In order to show how our approach can be used in this framework, note that the received signal $y(t)$ can be modeled as a convolution of $L$, possibly different, impulses with a known coding sequence $g(t)$, that is,

$$
y(t)=\sum_{l=1}^{L} a_{l} p_{l}\left(t-t_{l}\right) * g(t) .
$$

The DFT coefficients of $y(t)$ are now given by

$$
Y[n]=\sum_{l=1}^{L} a_{l} P_{l}[n] G[n] e^{-j n \omega_{c} t_{l}},
$$

where $\omega_{c}=2 \pi / T_{c}$, while $T_{c}$ denotes the cycle time. If we use the polynomial approximation of coefficients $P_{l}[n]$, the total number of degrees of freedom per cycle is $2 R L$, which determines the minimum sampling rate.

Another advantage of our solution is that it allows for a "multiresolution" or two-step approach, that is, one can first obtain a rough estimate of the sequence timing, by sampling the signal at a low rate over the entire cycle. Later, precise delay estimation can be carried out by increasing the sampling rate, yet sampling the received signal only within a narrow time window where it is present. The rationale for using the two-step approach is that a sequence duration $T_{s}$ typically spans a small fraction of the cycle time $T_{c}$ (e.g. less than 20\%), where search-based methods [2] [4], result in a long acquisition time and apparently "waste" power in processing time slots where the signal is not present. In the following, we will demonstrate the effectiveness of the two-step approach with a numerical example.

\section{SIMULATION RESULTS}

In this section, we show some simulation results that illustrate the performances of the proposed algorithms. We consider an UWB localization system, where a coded sequence of 127 UWB impulses is periodically transmitted, while the sequence duration spans approximately $20 \%$ of the cycle time $T_{c}$, as illustrated in Figure 1(a). We first consider the channel model (1), assuming $L=6$ paths with one dominant (containing $70 \%$ of the total power). In Figure 1(b), we show the root mean square error (RMSE) of time delay estimation for the dominant component. The results indicate that the method yields highly accurate estimates for a wide range of SNR's, and this with sub-Nyquist sampling rates. Note that the achievable RMSE with the approach using digital matched filters [2], is RMSE $=1$ (sample).

The two-step delay estimation method is analyzed next. During the coarse synchronization phase, the signal is sampled uniformly over the entire cycle at a low rate $N_{l}=0.05 N_{n}\left(N_{n}\right.$ is the Nyquist rate). For low values of SNR (that is, less than $-5 \mathrm{~dB}$ ), the samples are averaged over multiple cycles in order to increase the effective SNR. The second step is fine synchronization, where the signal is sampled at a higher rate $N_{h}=0.5 N_{n}$, yet within a narrow time window. RMSE of the two-step approach is shown in Figure 2(a). The error is compared to the RMSE obtained when the signal is sampled uniformly at a rate $0.5 N_{n}$ over the entire cycle. Clearly, the two solutions yield a similar performance, however, with the two-step approach, the computational requirements are 
reduced by a factor of 20 , while the power consumption reduces roughly by a factor of 3.3. In Figure 2(b), we plot the RMSE of delay estimation for different combinations of $N_{l}$ and $N_{h}$, assuming that the total number of cycles is 5 . The number of cycles during each phase is chosen such that the power consumption remains constant. Clearly, the performance improves as $N_{h}$ increases, yet at the expense of increased computational requirements.

We next consider the case of a channel model given by (1), assuming that a received signal is made up of $L=70$ pulses and 8 closely spaced dominant components, as shown in Fig. 3(a). In Fig. 3(b), we illustrate the effects of quantization on the estimation performance, specifically, we consider 4-7 bit architectures. Clearly, as the number of bits increases, the overall performance improves. Generally, the 5-bit architecture already yields a very good performance. Also note that when $n_{b} \geq 5$ and the value of SNR is low (e.g. $S N R<0 d B$ ), quantization has almost no impact on RMSE. However, as SNR increases, quantization noise becomes dominant and determines the overall performance.

\section{CONCLUSIONS}

We presented a frequency-domain framework for high-resolution parameter estimation in digital UWB receivers, which is based on our recent sampling results for certain parametric non-bandlimited signals. Our approach requires lower sampling rate and, therefore, lower complexity and power consumption compared to existing digital solutions. Besides, it leads to faster acquisition and allows for identification of more realistic channel models without resorting to complex algorithms. Our approach is particularly suitable in applications such as positioning or ranging, but can be used in other UWB applications as well, primarily for synchronization and channel characterization purposes.

\section{REFERENCES}

[1] R. J. Cramer, R. A. Scholtz and M. Z. Win, "Evaluation of an Ultra-Wideband Propagation Channel", IEEE Trans. on Antennas and Propagation, Vol. 50, No. 5, pp 561-570, May 2002.

[2] I. O' Donnell, M. Chen, S. Wang and R. Brodersen, "An Integrated, Low-Power, UWB Transceiver Architecture for Low-Rate Indoor Wireless System”, IEEE CAS Workshop on Wireless Communications and Networking, Sept. 2002.

[3] E. Homier and R. Scholtz, "Rapid Acquisition of UWB Signals in a Dense Multipath Channel", in Proc. IEEE Conf. on UWB Systems and Technologies, May 2002.

[4] R. Fleming, C. Kushner, G. Roberts and U. Nandiwada, "Rapid Acquisition for Ultra-Wideband Localizers", in Proc. IEEE Conf. on UWB Systems and Technologies, May 2002.

[5] I. Maravic, M. Vetterli and K. Ramchandran, "Highresolution acquisition methods for wideband communication systems", In Proc. of ICASSP, April 2003.

[6] I. Maravic, M. Vetterli and K. Ramchandran, "Highresolution methods for channel estimation and synchronization in UWB systems", IEEE Trans. on Signal Processing, submitted, 2003.

[7] B. D. Rao and K. S. Arun, "Model based processing of signals: A state space approach", Proceedings of the IEEE, Vol. 80, No. 2, pp. 283-309, February 1992.

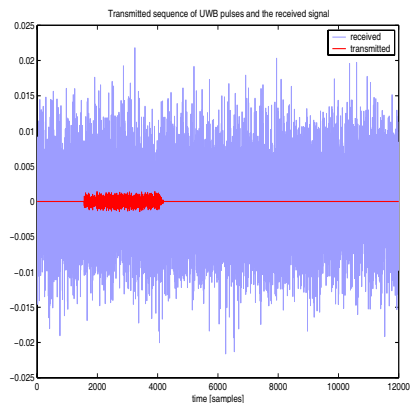

(a)

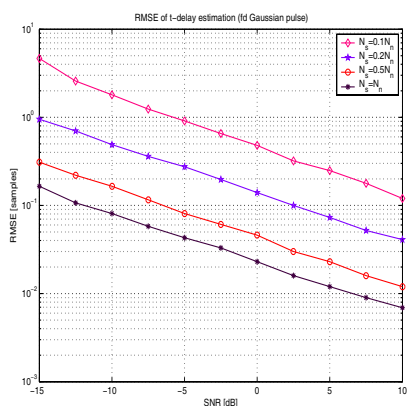

(b)
Fig. 1. Timing recovery in UWB systems (a) Received UWB sequence (red) and received signal during one cycle (blue). The transmitted pulse is an ideal first-derivative Gaussian impulse with the duration of (approximately) $T_{p}=5$ samples, while the spacing between the transmitted pulses is 20 samples. (b) Root-mean square error (RMSE) of delay estimation $v$ s. SNR (defined as the ratio between the power of the sequence and noise power in one cycle), and this for different sampling rates $N_{s} . N_{n}$ denotes the Nyquist rate.

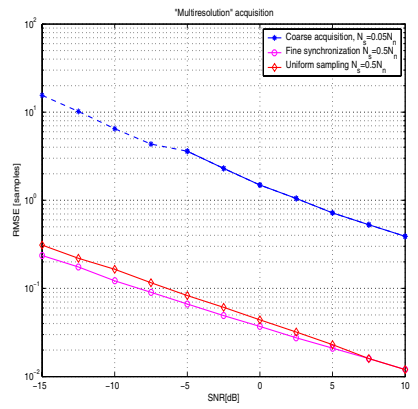

(a)

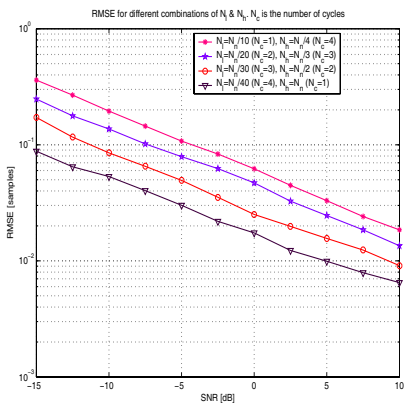

(b)
Fig. 2. Two-step delay estimation (a) RMSE of two-step time delay estimation is compared to the RMSE obtained with high-rate uniform sampling over the entire cycle. (b) RMSE of delay estimation for different combinations of $N_{l}$ (the sampling rate for coarse synchronization) and $N_{h}$ (the sampling rate for fine synchronization). $N_{c}$ denotes the number of averaging cycles during each phase.

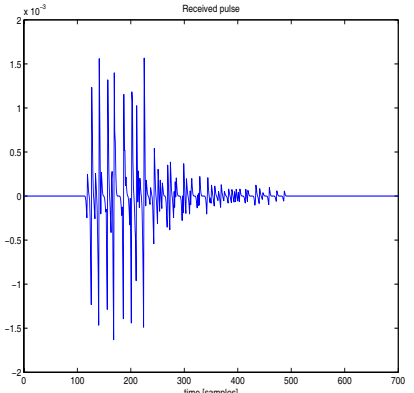

(a)

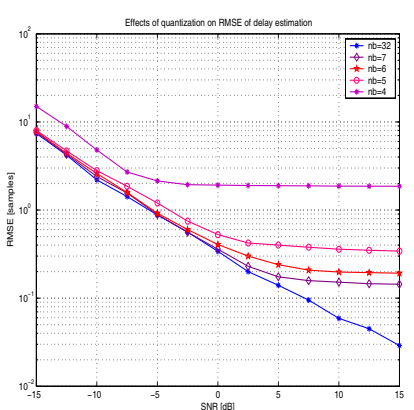

(b)
Fig. 3. Estimating higher-rank channel models (a) Received UWB signal made up of 70 pulses, with 8 components being dominant (containing approximately $85 \%$ of the total power). Average spacing between the received dominant components is $2 T_{p}$, where $T_{p}$ denotes the pulse duration. (b) Effects of quantization on the RMSE of delay estimation (dominant components only) for $4-7$ bit receiver architectures. The results are compared to the case when the number of bits is $n_{b}=32$. The sampling rate is one fourth the Nyquist rate $\left(N_{s}=N_{n} / 4\right)$. 